\title{
LXXXVI. Researches in the undulatory theory of light, in continuation of former papers
}

\author{
John Tovey Esq.
}

To cite this article: John Tovey Esq. (1836) LXXXVI. Researches in the undulatory theory of light, in continuation of former papers, Philosophical Magazine Series 3, 8:49, 500-505, DOI: 10.1080/14786443608648928

To link to this article: http://dx.doi.org/10.1080/14786443608648928

册 Published online: 01 Jun 2009.

Submit your article to this journal

ЏII Article views: 2

Q View related articles $\sqsubset$ 
existence of which as a constituent I could not determine at all, and which the quantities of the other constituents would appear absolutely to exclude. It is therefore necessary to examine whether any other method of arrangement is more satisfactory.

From the researches on oxamide, benzamide, \&c. it follows, that by the action of ammonia on an oxide there may be formed water and a compound of the body $\mathrm{N} \mathrm{H}^{2}$ with the base of the oxide. If we consider this to have taken place in white precipitate, we should have the formula

$$
\begin{aligned}
& (2 \mathrm{Cl}+\mathrm{Hg})+\left(2 \mathrm{~N} \mathrm{H}^{2}+\mathrm{Hg}\right) \text {, giving } \\
& 2 \mathrm{Hg}=405.60 \text { or, per cent. } 79.73 \\
& 2 \mathrm{Cl}=70.84 \longrightarrow \begin{array}{c}
13.93 \\
2 \mathrm{NH}^{z}=32.30
\end{array} \quad 6.34 ;
\end{aligned}
$$

and this compound should yield on analysis, on being decomposed, 6.73 per cent. of ammonia.

The question whether ammonia in acting on metallic oxides forms water and metallic amides is one of the most interesting now requiring to be examined; but notwithstanding the bearing the results just described have on the question, I do not wish to adopt too positively the opinion that white precipitate is a compound of deutochloride and deutamide of mercury until more extended researches shall enable me to argue from a greater number of facts derived from the reactions with other metals.

LXXXVI. Researches in the Undulatory Theory of Light, in continuation of former Papers. By JoHn Tovey, Esq.

To the Editors of the Philosophical Magazine and Journal. Gentuemen,

I NOW proceed, as I proposed at the conclusion of my last paper, p. 272, to integrate the equations

$$
\begin{aligned}
& \frac{d^{2} \xi}{d t^{2}}=s^{2} \cdot \frac{d^{2} \xi}{d x}+s^{\prime 2} \cdot \frac{d^{4} \xi}{d x^{4}}+\& c . \\
& \frac{d^{2} \eta}{d t^{2}}=s_{i}^{2} \cdot \frac{d^{2} \eta}{d x^{2}}+s_{i}^{12} \frac{d^{4} \eta}{d x^{4}}+\& \mathrm{c} . \\
& \frac{d^{2} \zeta}{d t^{3}}=s_{11}{ }^{2} \cdot d d^{2} \zeta x^{2}+s_{11}{ }^{12} \frac{d^{4} \zeta}{d x^{4}}+\& \mathrm{c} .
\end{aligned}
$$

As $\xi$ is, by supposition, a function of $x$ and $t$, we can put 


$$
\begin{aligned}
\xi & =p \sin k x+p^{\prime} \sin k^{\prime} x+p^{\prime \prime} \sin k^{\prime \prime} x+\& c . \\
& +q \cos k x+q^{\prime} \cos k^{\prime} x+q^{\prime \prime} \cos k^{\prime \prime} x+\& \mathrm{c} .
\end{aligned}
$$

where $p, p^{\prime}, p^{\prime \prime}, \& c ., q, q^{\prime}, q^{\prime \prime}, \&$ c. are functions of $t$, and $k, k^{\prime}, k^{\prime \prime}$, \&c. arbitrary constants. (Poisson, Traité de Mécanique, No. 514.) Now, if we substitute $p \sin k x+q \cos k x$ for $\xi$ in the first of the equations (1.), it becomes

$$
\begin{aligned}
& \left\{\frac{d^{2} p}{d t^{2}}+\left(\left(s^{2} k^{2}-s^{2} k^{4}+\& \mathrm{c} .\right) p\right\} \sin k x\right. \\
+ & \left\{\frac{d^{2} q}{d t^{2}}+\left(s^{2} k^{2}-s^{2 / 2} k^{4}+\& \mathrm{c} .\right) q\right\} \cos k x=0 ;
\end{aligned}
$$

and, this equation being true for all values of $x$, resolves itself into

$$
\begin{aligned}
& \frac{d^{2} p}{d t^{2}}+\left(s^{2} k^{2}-s^{2} k^{4}+\& c .\right) p=0 \\
& \frac{d^{2} q}{d t^{2}}+\left(s^{2} k^{2}-s^{\prime 2} k^{4}+8 c .\right) q=0 .
\end{aligned}
$$

The complete integrals of these equations are $p=A \sin n t$ $+\mathrm{B} \cos n t, q=\mathrm{A}^{\prime} \sin n t+\mathrm{B}^{\prime} \cos n t ; \quad$ where $\mathrm{A}, \mathrm{B}, \mathrm{A}^{\prime}, \mathrm{B}^{\prime}$, are arbitrary constants, and $n=\sqrt{ }\left(s^{8} k^{2}-s^{2} k^{4}+\& c_{\text {c. }}\right)$. Hence the complete integral of the first of the equations (1.) can be expressed by the sum of a series of functions, each of which is of the form

$(\mathrm{A} \sin n t+\mathrm{B} \cos n t) \sin k x+\left(\mathrm{A}^{\prime} \sin n t+\mathrm{B}^{\prime} \cos n t\right) \cos k x$.

This expression is, by the rules of trigonometry, equivalent to

$$
\begin{aligned}
& \frac{\mathrm{A}+\mathrm{B}^{\prime}}{2} \cos (n t-k x)+\frac{\mathrm{A}^{\prime}-\mathrm{B}}{2} \sin (n t-k x) \\
- & \frac{\mathrm{A}-\mathrm{B}^{\prime}}{2} \cos (n t+k x)+\frac{\mathrm{A}^{\prime}+\mathrm{B}}{2} \sin (n t+k x) ;
\end{aligned}
$$

which, if we put

$$
\begin{aligned}
\frac{\mathrm{A}+\mathrm{B}^{\prime}}{2}=\alpha \sin a, \frac{\mathrm{A}^{\prime}-\mathrm{B}}{2} & =\alpha \cos a, \frac{\mathrm{A}-\mathrm{B}^{\prime}}{2}=-\beta \sin b, \\
\frac{\mathrm{A}^{\prime}+\mathrm{B}}{2} & =\beta \cos b,
\end{aligned}
$$

may, by the same rules, be reduced to

$$
\alpha \sin (n t-k x+a)+\beta \sin (n t+k x+b) \text {. }
$$

It follows, therefore, that since the second and third of the equations (1.) are of the same form as the first, and since $\eta$ and $\zeta$ are, like 1 , functions of $x$ and $t$, we can express the complete integrals of these equations by putting 


$$
\begin{aligned}
& \xi=\Sigma \cdot \alpha \sin (n t-k x+a)+\Sigma \cdot \beta \sin (n t+k x+b), \\
& \eta=\Sigma \cdot \alpha_{1} \sin \left(n, t-k, x+a_{\jmath}\right)+\Sigma \cdot \beta, \sin (n, t+k, x+b), \\
& \zeta=\Sigma \cdot \alpha_{l l} \sin \left(n_{t l} t-k_{l l} x+a_{l l}\right)+\Sigma \cdot \beta_{i l} \sin \left(n_{b l} t+k_{l l} x+b_{l l}\right)
\end{aligned}
$$

where

$$
\begin{aligned}
& n=\sqrt{ }\left(s^{2} k^{2}-s^{2} k^{4}+\& \mathrm{c} .\right), \\
& n_{l}=\sqrt{ }\left(s_{l}^{2} k_{l}^{2}-s_{i}^{\prime 2} k_{l}^{4}+\& c_{0}\right) \text {, } \\
& n_{u}=\mathcal{V}\left(s_{11}{ }^{2} k_{u l}{ }^{2}-s_{u}^{\prime}{ }^{8} k_{u}{ }^{4}+\& \mathrm{c} .\right):
\end{aligned}
$$

the sums $\Sigma$ being extended to all the requisite values of the arbitrary constants.

We perceive, by the equations (2.), that the motion of the system may be regarded as compounded of a number of coexisting movements, severally expressed by the terms of the sums $\Sigma$. And when we confine our attention to a single term of the first sum in one of these equations, which we may do in a great variety of problems, we have virtually the same expression for the displacement of a molecule of an undulating medium, as is assumed tacitly by Sir Isaac Newton, and expressly by Professor Airy*.

Taking separately the displacement $\eta$, and considering only one term of the first sum in the expression for this quantity, we have

$$
\eta=a_{1} \sin \left(n_{1} t-k_{1} x+a_{1}\right) .
$$

It is well known that this equation represents a series of equal and continuous waves, the length of each wave being $\frac{2 \pi}{k}$; where $2 \pi$ is the circumference of a circle whose radius is unity. Now, if we increase $x$ uniformly, so as to make $n_{1} t-k_{1} x$ constant, $\eta$ remains constant, and $\frac{d x}{d t}=\frac{n_{l}}{k_{l}}$. Hence we perceive that these waves travel, in the direction of $x$ positive, with a velocity equal to $\frac{n_{i}}{k_{l}}$. If $\eta=\beta, \sin (n, t$ $\left.+k_{i} x+b_{1}\right)$, which is a term of the second sum, the movement is similar, except that the waves travel in the contrary direction.

The second of the equations (3.) gives

$$
\frac{n_{1}}{k_{l}}=s_{i} \sqrt{ }\left(1-\frac{s_{1}^{\prime 2}}{s_{i}^{2}} k_{i}^{2}+\& \mathbf{c} .\right) \text {; }
$$

an equation affording the same theory of dispersion as that which has been so satisfactorily investigated and verified by

- See Airy's Mathemat. Tracts, p. 255. 
Professor Powell in the recent Numbers of your Journal. This I have shown more explicitly in your Number for January last, p. 7 .

Since, by the last equation, the velocity of the waves, and consequently the refraction of the light at the surface of the medium, depends chiefly upon $s_{l}$, while the dispersion depends upon $\frac{s_{1}^{\prime 2}}{s_{1}^{2}} k_{1}^{2}$ and the following terms of the series, we see that the dispersion may be different for different media, though the mean refraction be the same; contrary to the opinion which so long retarded the improvement of refracting telescopes.

The equations (3.) may, perhaps, lead to a theory of absorption as well as of dispersion; since it is obvious that they may become impossible for particular values of $k$. It should be observed that the sums $s^{2}, s_{1}^{2}, s_{u}^{2}, s^{2}$, \&c. are not necessarily positive, and I now think it would be better to denote them by $s, s_{l}, s_{l,}, s^{\prime}, \& c$. I adopted the other notation in order to assimilate the formulæ to those employed in the theory of sound.

In the case of undulation which we have been considering, the waves are plane waves, perpendicular to the axis of $x$; we now pass on to the consideration of converging and diverging waves.

Let us take the case of a system of waves going and returning to and from a certain point; calling this point the centre of agitation. Then the diameter of the sphere of influence of any molecule being an insensible quantity, it is evident that the minute portion of one of the waves contained within the sphere cannot, at any sensible distance from the centre of agitation, differ sensibly from the same portion of a plane wave. Therefore, as the motion of any molecule is. affected only by the molecules within the sphere of its influence, it follows that the equations (3.), which give the velocities $\frac{n}{k}, \frac{n_{i}}{k_{l}}, \frac{n_{l}}{k_{l l}}$, of plane waves, will also give, at any point of the system, the velocities with which diverging or converging waves are transmitted in the direction perpendicular to the wave-surface at that point.

When the molecules are so arranged that the sums $s^{2}, s_{i}^{2}$, $s_{11}{ }^{2}$, \&c. are the same for all directions of the rectangular coordinates, the velocities of the waves are the same for every radius drawn from the centre of agitation; and consequently the wave-surfaces are spherical. 
If we conceive a slowly tapering cone (fig. 1.) to have its

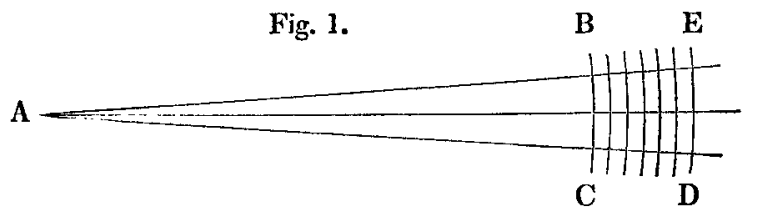

summit $A$ at the centre of agitation of a system of spherical waves, and if we take the axis of the cone for the axis of $x$, it is clear that the displacements $\xi, \eta$, $\zeta$, of the molecules within the frustum B C D E may be regarded as functions of $x$ and $t$; and may therefore be expressed by the equations (2.), nearly. It is also manifest that the same equations will express the displacements for any other frustum of the medium, by making the arbitrary quantities to vary according to the position of the frustum. Consequently, if we suppose

$$
\xi=\alpha \sin (n t-k x+a)
$$

for the frustum B C D E, the same equation may be taken to express the value of $\xi$ for any other frustum of the same cone, by regarding $a, n, k, a$ as functions of $x$.

Let $\rho$ be the radius of the sphere of influence of the molecules: then, if $\frac{\rho}{x}$ were infinitely small, the minute portion of a wave contained within the sphere would be a plane wave, and $\alpha, n, k, a$ constant. Hence we perceive that these quantities must be functions of $\frac{\rho}{x}$; and consequently, that we may write

$$
\begin{aligned}
& \alpha=\mathrm{A}+\mathrm{B} \frac{\rho}{x}+\mathrm{C}\left(\frac{\rho}{x}\right)^{2}+\& \mathrm{c} ., \\
& n=\mathrm{A}^{\prime}+\mathrm{B}^{\prime} \frac{\rho}{x}+\mathrm{C}^{\prime}\left(\frac{\rho}{x}\right)^{2}+\& \mathrm{c}, \\
& k=\mathrm{A}^{\prime \prime}+\mathrm{B}^{\prime \prime} \frac{\rho}{x}+\mathrm{C}^{\prime \prime}\left(\frac{\rho}{x}\right)^{2}+\& \mathrm{c} . \\
& a=\mathrm{A}^{\prime \prime \prime}+\mathrm{B}^{\prime \prime \prime} \frac{\rho}{x}+\mathrm{C}^{\prime \prime \prime}\left(\frac{\rho}{x}\right)^{2}+\& \mathrm{c} .:
\end{aligned}
$$

the only variable quantity in these series being $x$.

Now when $x$ is infinite $\alpha$ must be zero; therefore $\mathrm{A}=0$ : and as $\frac{\rho}{x}$ is, at all sensible distances from the centre of agi- 
tation, an extremely small quantity, we may reject its powers above the first; therefore $a=\mathrm{B} \frac{\rho}{x}$. The quantities $n, k, a$ approach, as $\boldsymbol{x}$ increases, towards the values which they have in the case of plane waves, which values are independent of $\alpha$. And since the small portion of a wave contained within the sphere of influence of any molecule cannot, at any sensible distance from the centre of agitation, differ sensibly from the same portion of a plane wave, we may regard $n, k, a$ as constant for all parts of the cone. If then we retain $\alpha$ to denote $\mathrm{B}_{\xi}$, the constant part of $\frac{\mathrm{B}_{\rho}}{x}$, we have

$$
\xi=\frac{a}{x} \sin (n t-k x+a):
$$

and, in general, for any cone taken as we have supposed, we have, from the equations (2.),

$$
\begin{aligned}
& \xi=\Sigma \cdot \frac{\alpha}{x} \sin (n t-k x+a)+\Sigma \cdot \frac{\beta}{x}(\sin n t+k x+b), \\
& \eta=\Sigma \cdot \frac{\alpha}{x} \sin \left(n, t-k_{1}, x+a_{1}\right)+\Sigma \cdot \frac{\beta_{1}}{x} \sin (n, t+k, x+b),(4 \cdot) \\
& \zeta=\Sigma \cdot \frac{\alpha_{i 1}}{x} \sin \left(n_{\| 1} t-k_{i l} x+a_{\| \prime}\right)+\Sigma \cdot \frac{\beta_{\| \prime}}{x} \sin \left(n_{\| l} t+k_{\| l} x+b_{u}\right) \text {. }
\end{aligned}
$$

When the waves all move from the centre of agitation, the second sums in the equations (4.) will vanish: and limiting our view to a single term of one of the first sums, we have an expression for the displacement virtually the same as that which Professor Airy, in his valuable tract on the Undulatory Theory of Optics, has partly assumed and partly borrowed from the theory of sound*.

It may be observed, by the way, that the method adopted in this paper of expressing the displacement of the molecules, is analogous to that employed so successfully in physical astronomy to express the differences between the mean and true places of the planets.

When the molecules are so arranged that the sums $s^{2}, s^{2}$, $s_{u l}^{2}$, \&c. are different for different directions of the coordinates, waves going and returning to and from a centre of agitation will not be spherical. The most simple case of such waves will probably furnish a subject for another paper.

Evesham, April 15, 1836.

$$
\text { I am, Gentlemen, yours, \&c. }
$$

P.S. I perceive that throughout my last paper I inadvertently called the differences $\Delta x, \Delta y, \Delta z$ variations.

$$
\text { * Mathenat. Tracts, p. } 271 \text {. }
$$

Third Series. Vol. 8. No. 49. June 1836. 